\title{
Optimal Dividend and Capital Injection Strategies for a Risk Model under Force of Interest
}

\author{
Ying Fang ${ }^{1}$ and Zhongfeng $Q \mathbf{u}^{2}$ \\ ${ }^{1}$ School of Mathematical Sciences, Shandong Normal University, Jinan 250014, China \\ ${ }^{2}$ School of Mathematical Sciences, University of Jinan, Jinan 250022, China \\ Correspondence should be addressed to Zhongfeng Qu; quzhongfeng79@163.com
}

Received 30 January 2013; Accepted 14 April 2013

Academic Editor: Guangchen Wang

Copyright (C) 2013 Y. Fang and Z. Qu. This is an open access article distributed under the Creative Commons Attribution License, which permits unrestricted use, distribution, and reproduction in any medium, provided the original work is properly cited.

As a generalization of the classical Cramér-Lundberg risk model, we consider a risk model including a constant force of interest in the present paper. Most optimal dividend strategies which only consider the processes modeling the surplus of a risk business are absorbed at 0 . However, in many cases, negative surplus does not necessarily mean that the business has to stop. Therefore, we assume that negative surplus is not allowed and the beneficiary of the dividends is required to inject capital into the insurance company to ensure that its risk process stays nonnegative. For this risk model, we show that the optimal dividend strategy which maximizes the discounted dividend payments minus the penalized discounted capital injections is a threshold strategy for the case of the dividend payout rate which is bounded by some positive constant and the optimal injection strategy is to inject capitals immediately to make the company's assets back to zero when the surplus of the company becomes negative.

\section{Introduction}

In the mathematical finance and the actuarial literature, the optimal dividend problem has attracted much attention. For example, there is a good deal of work on the problem of finding an optimal policy for paying out dividends. In the Brownian motion setting, it has been proved that the optimal dividend strategy is a threshold strategy for the case of the dividend payout rate is which bounded by some positive constant and a barrier strategy for the case with no restriction on the dividend payout rate by Asmussen and Taksar [1]. In the Cramér-Lundberg setting, it was first shown in [2] by a discrete approximation and then a limiting argument that the optimal dividend strategy is of the so-called band type. For exponentially distributed claim sizes this strategy simplifies to a barrier strategy. This result was rederived by stochastic control theory in $[3,4]$. Recently, Albrecher and Thonhauser [5] studied the optimal dividend strategy by viscosity theory in the constant force of interest model. They pointed out that the optimal dividend strategy in the general case is again of band type and for exponential claim sizes collapses to a barrier strategy. In addition, Avram et al. [6] and Loeffen
[7] considered the optimal dividend problem when the risk process is modeled by a spectrally negative Lévy process. They drew on the fluctuation theory of spectrally negative Lévy processes and gave sufficient conditions under which the optimal strategy is of barrier type.

Unfortunately, a drawback is that processes modeling the surplus of a risk business are absorbed at 0 . In many cases, negative surplus does not necessarily mean that the business has to stop. Therefore, many authors suggested a model, where the above-mentioned fact should be taken into account.

One method is to make 0 as a reflecting barrier rather than absorbing barrier. For example, Shreve et al. [8] proposed that a diffusion process can be controlled by subtracting out a withdrawal (dividend) process and adding in a deposit (injection) process. The goal is then to maximize the expected discounted dividend payments minus the expected penalized discounted capital injections. If the surplus process is a Brownian motion with drift, they found that the optimal injection policy is to invest the minimum such that the controlled surplus remains positive. Furthermore, they also show that the optimal dividend policy is a barrier strategy. 
Løkka and Zervos [9] added proportional costs to the deposits. In the more general framework of spectrally negative Lévy processes, Avram et al. [6] studied the optimality of barrier strategies with capital injections. In the CramérLundberg model, Kulenko and Schmidli [10] showed that the optimal dividend strategy is a barrier strategy and the optimal injection policy is to inject the minimum such that the controlled surplus remains nonnegative. In this paper, we will solve the problem for a risk process under a constant force of interest.

Consider the following risk model for the reserve process $\{U(t)\}$ of an insurance portfolio:

$$
U_{t}=x e^{r t}+c \int_{0}^{t} e^{r(t-s)} d s-\int_{0}^{t} e^{r(t-s)} d \sum_{i=1}^{N_{s}} X_{i}
$$

where $x \geq 0$ is the initial capital, $c>0$ is the premium rate, $r>$ 0 is the constant force of interest, $\left\{N_{t}, t \geq 0\right\}$ is a homogeneous Poisson claim counting process with intensity $\lambda>0$, and $\left\{X_{i}, i=1,2, \ldots\right\}$ are i.i.d. claim size random variables, which are independent of $\left\{N_{t}, t \geq 0\right\}$ and have common distribution function $P(\cdot)$ with $P(0)=0$, density function $p(\cdot)$, and mean $E\left[X_{i}\right]=\mu$. Let $\pi$ denote an admissible strategy consisting of an accumulated dividend process $\left\{D_{t}^{\pi}\right\}$ and an accumulated injection process $\left\{Z_{t}^{\pi}\right\}$. The accumulated dividend process $\left\{D_{t}^{\pi}\right\}$ is a nondecreasing and left continuous process with $D_{0}^{\pi}=0$; the accumulated injection process $\left\{Z_{t}^{\pi}\right\}$ is a nondecreasing and right continuous process with $Z_{0-}^{\pi}=$ 0 . Then the surplus process modified by policy $\pi$ becomes

$$
U_{t}^{\pi}=U_{t}-\int_{0}^{t} e^{r(t-s)} d D_{s}^{\pi}+\int_{0}^{t} e^{r(t-s)} d Z_{s}^{\pi} .
$$

The set of admissible policies $\Pi$ consists of those policies which satisfy

(i) $U_{t}^{\pi}$ is nonnegative for $t>0$,

(ii) $\int_{0}^{\infty} e^{-\delta t} d Z_{t}^{\pi}<\infty$ almost surely.

The value associated to the strategy $\pi$ is defined as

$$
V_{\pi}(x)=E\left[\int_{0}^{\infty} e^{-\delta t} d D_{t}^{\pi}-\kappa \int_{0}^{\infty} e^{-\delta t} d Z_{t}^{\pi} \mid U_{0}^{\pi}=x\right],
$$

where $\delta>0$ is the force of interest for discounting the dividends and $\kappa$ is the cost per unit injected capital or a penalizing factor. In this paper, we assume that $\delta>r$ and $\kappa>1$. The maximal value function is denoted by

$$
V(x)=\sup _{\pi \in \Pi} V_{\pi}(x) .
$$

Let us illustrate the reason why we choose $\kappa>1$. If we had chosen $\kappa<1$, the maximal value function would be infinite because it is cheaper to pay dividends by injection capital than from the reserve. If we had chosen $\kappa=1$, the cost of paying incoming claims from the reserve is the same as by an injection capital. However, the discount factor $\delta$ is greater than the force of interest $r$. Then it is better to pay dividends earlier. Therefore, it is optimal to pay out all positive surplus immediately as dividends and to pay all claims by injection capital. That is,

$$
\begin{aligned}
V(x) & =x+\int_{0}^{\infty} e^{-\delta t} c d t-E\left[\sum_{i=1}^{\infty} e^{-\delta T_{i}} X_{i}\right] \\
& =x+\frac{c-\lambda \mu}{\delta}
\end{aligned}
$$

where $T_{k}$ is the time of the $k$ th claim.

In addition, notice that from (3) it can not be optimal to inject capital before they are really necessary because of $\kappa>1$ and $\delta>r$. Therefore, we only need to choose the dividend strategy $\left\{D_{t}^{\pi}\right\}$. The corresponding injection process $\left\{Z_{t}^{D^{\pi}}\right\}$ is defined as follows: when the surplus of the company with strategy $\left\{D_{t}^{\pi}\right\}$ becomes negative, the shareholders immediately inject the amount of the deficit such that the surplus can be restored to 0 ; when the surplus is nonnegative, no capitals are injected. If the initial capital is negative, then $Z_{0}=|x|$, thus $V(x)=V(0)-\kappa|x|$.

In this paper, we impose restriction on the dividend stream to prevent ruin occurring almost surely. We assume that the dividends are paid at a dynamic rate $d_{t}$ at time $t$. Stochastic process $\left\{d_{t}\right\}$ is called a control process and can only vary in $[0, \alpha]$ for some $0<\alpha<c$. This optimal dividend problem with additional constraints is postulated by JeanblancPicqué and Shiryaev [11] and Asmussen and Taksar [1].

The outline of the remainder of the paper is organized as follows. In Section 2, we prove some properties of the maximal dividend-value function and derive the HamiltonJacobi-Bellman equation for the problem. In Section 3, we give the optimal strategy by the HJB equation and the concavity of the value function. We show that the optimal dividend strategy is a threshold strategy. Some concluding remarks are given in Section 4.

\section{The Maximal Value Function}

We first prove some properties of the maximal dividend-value function.

Proposition 1. The maximal value function $V(x)$ is increasing and converges to $\alpha / \delta$ as $x$ converges to $\infty$.

Proof. Let $\pi_{\varepsilon}^{x}$ be an $\varepsilon$-optimal strategy for initial capital $x$, that is, $V_{\pi_{\varepsilon}^{x}}(x) \geq V(x)-\varepsilon$. Let $\left\{d_{t}^{\pi_{\varepsilon}^{x}}\right\}$ be the corresponding dividend rate process. For initial capital $y(y>x)$, we define a policy $\pi^{y}$ with dividend rate

$$
d_{t}^{\pi^{y}}=d_{t}^{\pi_{\varepsilon}^{x}}
$$

Note that this strategy is admissible for initial capital $y$ and its injection process has the following relationship with $Z^{d^{\pi_{\varepsilon}^{x}}}$ :

$$
Z_{t}^{d^{\pi^{y}}} \leq Z_{t}^{d^{\pi_{\varepsilon}^{x}}}
$$


Hence we have

$$
\begin{aligned}
V(y) & \geq E\left[\int_{0}^{\infty} e^{-\delta t} d_{t}^{\pi^{y}} d t-\kappa \int_{0}^{\infty} e^{-\delta t} d Z_{t}^{d^{\pi^{y}}}\right] \\
& \geq E\left[\int_{0}^{\infty} e^{-\delta t} d_{t}^{\pi_{\varepsilon}^{x}} d t-\kappa \int_{0}^{\infty} e^{-\delta t} d Z_{t}^{d^{\pi_{\varepsilon}^{x}}}\right] \\
& \geq V(x)-\varepsilon .
\end{aligned}
$$

Since this inequality holds for all $\varepsilon>0$, then we have $V(y) \geq$ $V(x)$.

Consider a strategy $\hat{\pi}$ with $d_{t}^{\hat{\pi}}=\alpha$. Then the time of the first injection under this strategy

$$
\begin{aligned}
T_{\widehat{\pi}}=\inf \{t & \geq 0: x e^{r t}+\int_{0}^{t} e^{r(t-s)}(c-\alpha) d s \\
& \left.-\int_{0}^{t} e^{r(t-s)} d S_{s}<0\right\}
\end{aligned}
$$

converges to infinity as $x \rightarrow \infty$. Recall that capitals are injected only if the surplus process is below 0 , then

$$
\begin{aligned}
& E \int_{0}^{\infty} e^{-\delta t} d Z_{t}^{\hat{\pi}} \\
& \quad \leq E\left\{e^{-\delta T_{\hat{\pi}}}\left|U_{T_{\hat{\pi}}}^{\hat{\pi}}\right|+\sum_{i=N_{T_{\hat{\pi}}}+1}^{\infty} e^{-\delta T_{i}} X_{i}\right\} \\
& \quad=E\left\{e^{-\delta T_{\hat{\pi}}}\left[\left|U_{T_{\hat{\pi}}}^{\hat{\pi}}\right|+\sum_{i=N_{T_{\hat{\pi}}}+1}^{\infty} e^{-\delta\left(T_{i}-T_{\hat{\pi}}\right)} X_{i}\right]\right\} \\
& \quad=E\left\{e^{-\delta T_{\hat{\pi}}}\left|U_{T_{\hat{\pi}}^{\hat{\pi}}}\right|\right\}+E\left\{e^{-\delta T_{\hat{\pi}}}\left[\sum_{i=N_{T_{\hat{\pi}}}+1}^{\infty} e^{-\delta\left(T_{i}-T_{\hat{\pi}}\right)} X_{i}\right]\right\} \\
& \quad=E\left\{e^{-\delta T_{\hat{\pi}}}\left|U_{T_{\hat{\pi}}}^{\hat{\pi}}\right|\right\}+E\left[e^{-\delta T_{\hat{\pi}}}\right] E\left[\sum_{i=1}^{\infty} e^{-\delta T_{i}} X_{i}\right] \\
& \quad \leq E\left\{e^{-\delta T_{\hat{\pi}}}\left|U_{T_{\hat{\pi}}}^{\hat{\pi}}\right|\right\}+\frac{\lambda \mu}{\delta} E\left[e^{-\delta T_{\hat{\pi}}}\right] .
\end{aligned}
$$

Note that

$$
\begin{gathered}
\lim _{x \rightarrow \infty} E\left[e^{-\delta T_{\hat{\pi}}} \mid U_{0}^{\hat{\pi}}=x\right]=0, \\
\lim _{x \rightarrow \infty} E\left\{e^{-\delta T_{\hat{\pi}}}\left|U_{T_{\hat{\pi}}}^{\hat{\pi}}\right| \mid U_{0}^{\hat{\pi}}=x\right\}=0 .
\end{gathered}
$$

So,

$$
\lim _{x \rightarrow \infty} E\left[\int_{0}^{\infty} e^{-\delta t} d Z_{t}^{\hat{\pi}} \mid U_{0}^{\hat{\pi}}=x\right]=0
$$

Thus, as $x \rightarrow \infty$,

$$
V_{\widehat{\pi}}(x)=E\left[\int_{0}^{\infty} e^{-\delta t} \alpha d t-\kappa \int_{0}^{\infty} e^{-\delta t} d Z_{t}^{\widehat{\pi}} \mid U_{0}^{\widehat{\pi}}=x\right] \rightarrow \frac{\alpha}{\delta} .
$$

Naturally, it holds that

$$
V_{\widehat{\pi}}(x) \leq V(x) \leq \frac{\alpha}{\delta} .
$$

Hence, by squeeze theorem, we obtain

$$
\lim _{x \rightarrow \infty} V(x)=\frac{\alpha}{\delta}
$$

Proposition 2. The maximal value function $V(x)$ is concave and Lipschitz continuous.

Proof. Consider $x \geq 0, y \geq 0$, and $\theta \in(0,1)$. Let $\pi^{x}=$ $\left(d^{x}, Z^{d^{x}}\right)$ be an admissible strategy for the initial capital $x$ and let $\pi^{y}=\left(d^{y}, Z^{d^{y}}\right)$ be an admissible strategy for the initial capital $y$. We define strategy $\left(\theta d^{x}+(1-\theta) d^{y}, \theta Z^{d^{x}}+(1-\theta) Z^{d^{y}}\right)$ and find that

$$
\begin{aligned}
& {[\theta x+(1-\theta) y]+\int_{0}^{t} e^{r(t-s)} c d s-\int_{0}^{t} e^{r(t-s)} d \sum_{i=1}^{N_{s}} X_{i}} \\
& -\int_{0}^{t} e^{r(t-s)}\left[\theta d_{s}^{x}+(1-\theta) d_{s}^{y}\right] d s \\
& +\int_{0}^{t} e^{r(t-s)} d\left[\theta Z_{s}^{d^{x}}+(1-\theta) Z_{s}^{d^{y}}\right] \\
& =\theta\left\{x+\int_{0}^{t} e^{r(t-s)}\left(c-d_{s}^{x}\right) d s-\int_{0}^{t} e^{r(t-s)} d \sum_{i=1}^{N_{s}} X_{i}\right. \\
& \left.+\int_{0}^{t} e^{r(t-s)} d Z_{s}^{d^{x}}\right\} \\
& +(1-\theta)\left\{y+\int_{0}^{t} e^{r(t-s)}\left(c-d_{s}^{y}\right) d s\right. \\
& -\int_{0}^{t} e^{r(t-s)} d \sum_{i=1}^{N_{s}} X_{i} \\
& \left.+\int_{0}^{t} e^{r(t-s)} d Z_{s}^{d^{y}}\right\} \geq 0
\end{aligned}
$$

which implies that policy $\left(\theta d^{x}+(1-\theta) d^{y}, \theta Z^{d^{x}}+(1-\theta) Z^{d^{y}}\right)$ is an admissible dividend strategy for the initial capital $\theta x+$ $(1-\theta) y$. Denote $\theta d^{x}+(1-\theta) d^{y}=d^{\theta x+(1-\theta) y}$ and let $Z^{d^{\theta x+(1-\theta) y}}$ be the corresponding injection process. Then we must have $Z_{t}^{d^{\theta x+(1-\theta) y}} \leq \theta Z_{t}^{d^{x}}+(1-\theta) Z_{t}^{d^{y}}$. Therefore, it follows that

$$
\begin{array}{r}
V(\theta x+(1-\theta) y) \geq E\left[\int_{0}^{\infty} e^{-\delta t} d_{t}^{\theta x+(1-\theta) y} d t\right. \\
\left.-\kappa \int_{0}^{\infty} e^{-\delta t} d Z_{t}^{d^{\theta x+(1-\theta) y}}\right]
\end{array}
$$




$$
\begin{aligned}
& \geq E\left[\int_{0}^{\infty} e^{-\delta t}\left[\theta d_{t}^{x}+(1-\theta) d_{t}^{y}\right] d t\right. \\
& \left.\quad-\kappa \int_{0}^{\infty} e^{-\delta t} d\left[\theta Z_{t}^{d^{x}}+(1-\theta) Z_{t}^{d^{y}}\right]\right] \\
& =\theta V^{d^{x}}(x)+(1-\theta) V^{d^{y}}(y) .
\end{aligned}
$$

Taking the supremum over all admissible strategies in $\Pi$, we find that

$$
V(\theta x+(1-\theta) y) \geq \theta V(x)+(1-\theta) V(y) .
$$

Then the concavity of the maximal value function $V(x)$ follows.

Let $\pi_{y}=\left\{d_{t}^{y}, Z_{t}^{d^{y}}\right\}$ be an arbitrary admissible strategy for initial capital $y$. Let $h$ denote the time that the surplus reaches $y$ from $x$ if no claims occur, that is,

$$
h=\frac{1}{r} \ln \frac{c+r y}{c+r x} .
$$

For initial capital $x$, consider a dividend strategy

$$
d_{t}^{x}= \begin{cases}0, & \text { for } t \leq h \text { or } T_{1} \leq h, \\ d_{t-h}^{y}, & \text { for } T_{1} \wedge t>h .\end{cases}
$$

Then the probability that there is dividend payment is the probability that the surplus reaches $y$ without claims occurring first. This probability is $e^{-\lambda h}$. So, we have

$$
\begin{aligned}
& V(x) \\
& \geq E\left[\int_{0}^{\infty} e^{-\delta t} d_{t}^{x} d t-\kappa \int_{0}^{\infty} e^{-\delta t} d Z_{t}^{d^{x}}\right] \\
& =P\left(T_{1}>h\right) E\left[\int_{0}^{\infty} e^{-\delta t} d_{t}^{x} d t-\kappa \int_{0}^{\infty} e^{-\delta t} d Z_{t}^{d^{x}} \mid T_{1}>h\right] \\
& \quad+P\left(T_{1} \leq h\right) E\left[\int_{0}^{\infty} e^{-\delta t} d_{t}^{x} d t-\kappa \int_{0}^{\infty} e^{-\delta t} d Z_{t}^{d^{x}} \mid T_{1} \leq h\right] \\
& =e^{-\lambda h} e^{-\delta h} V_{\pi_{y}}(y)-\left(1-e^{-\lambda h}\right) E\left[\kappa \int_{0}^{\infty} e^{-\delta t} d Z_{t}^{d^{x}} \mid T_{1} \leq h\right] \\
& \geq e^{-(\lambda+\delta) h} V_{\pi_{y}}(y)-\kappa\left(1-e^{-\lambda h}\right) E\left[\sum_{i=1}^{\infty} e^{-\delta T_{i}} X_{i}\right] \\
& =e^{-(\lambda+\delta) h} V_{\pi_{y}}(y)-\frac{\kappa \lambda \mu}{\delta}\left(1-e^{-\lambda h}\right) .
\end{aligned}
$$

Taking the supremum over all $\Pi$ yields

$$
V(x) \geq e^{-(\lambda+\delta) h} V(y)-\frac{\kappa \lambda \mu}{\delta}\left(1-e^{-\lambda h}\right) .
$$

Note that

$$
\begin{gathered}
1-e^{-(\lambda+\delta) h} \leq(\lambda+\delta) h \leq \frac{\lambda+\delta}{c}(y-x), \\
1-e^{-\lambda h} \leq \lambda h \leq \frac{\lambda}{c}(y-x) .
\end{gathered}
$$

Thus, by (22) and (23), we obtain

$$
\begin{aligned}
0 \leq V(y)-V(x) & \leq\left(1-e^{-(\lambda+\delta) h}\right) V(y)+\frac{\kappa \lambda \mu}{\delta}\left(1-e^{-\lambda h}\right) \\
& \leq \frac{(\lambda+\delta) \alpha+\kappa \lambda^{2} \mu}{c \delta}(y-x),
\end{aligned}
$$

which implies that Lipschitz continuity holds. Therefore, $V(x)$ is absolutely continuous.

Lemma 3. The maximal value function $V(x)$ is differentiable from the right, and the right derivative $V^{\prime}(x+)$ satisfies $H J B$ equation:

$$
\begin{aligned}
\max _{0 \leq d \leq \alpha}\left\{(c-d+r x) V^{\prime}(x+)-(\lambda+\delta) V(x)\right. \\
\left.+\lambda \int_{0}^{\infty} V(x-y) p(y) d y+d\right\}=0 .
\end{aligned}
$$

Proof. Take a constant $u \in[0, \alpha]$. Let $h>0$ be small enough and $\varphi(x, h)=x e^{r h}+\int_{0}^{h} e^{r(h-s)}(c-u) d s$. We can show analogously to the proof of Proposition 3.1 of $[3,12,13]$ that the maximal dividend-value function fulfills dynamic programming principle for any stopping times $\tau$ :

$$
V(x)=\sup _{\pi \in \Pi} E\left[\int_{0}^{\tau \wedge T_{\pi}} e^{-\delta t} d_{t} d t+e^{-\delta\left(\tau \wedge T_{\pi}\right)} V\left(U_{\tau \wedge T_{\pi}}^{\pi}\right)\right] .
$$

Taking $\tau=T_{1} \wedge h$ in (26), we derive

$$
\begin{aligned}
V(x)= & \sup _{\pi \in \Pi} E\left[\int_{0}^{T_{1} \wedge h} e^{-\delta t} d_{t} d t+e^{-\delta\left(T_{1} \wedge h\right)} V\left(U_{T_{1} \wedge h}^{\pi}\right)\right] \\
\geq & e^{-\lambda h}\left[\int_{0}^{h} e^{-\delta t} u d t+e^{-\delta h} V(\varphi(x, h))\right] \\
& +\int_{0}^{h} \lambda e^{-\lambda t}\left[\int_{0}^{t} e^{-\delta s} u d s\right. \\
& \left.+e^{-\delta t} \int_{0}^{\infty} V(\varphi(x, t)-y) p(y) d y\right] d t
\end{aligned}
$$

Rearranging the terms and dividing by $h$ yields

$$
\begin{aligned}
& \frac{V(\varphi(x, h))-V(x)}{h}-\frac{1-e^{-(\lambda+\delta) h}}{h} V(\varphi(x, h)) \\
& +\frac{e^{-\lambda h}}{h} \int_{0}^{h} e^{-\delta t} u d t \\
& +\frac{1}{h} \int_{0}^{h} \lambda e^{-\lambda t}\left[\int_{0}^{t} e^{-\delta s} u d s\right. \\
& \left.+e^{-\delta t} \int_{0}^{\infty} V(\varphi(x, t)-y) p(y) d y\right] d t \leq 0 .
\end{aligned}
$$


On the other hand, from the dynamic programming principle, there exists a strategy $\pi^{h^{2}}=\left\{d_{t}^{h^{2}}\right\}$ such that

$$
E\left[\int_{0}^{T_{1} \wedge h} e^{-\delta t} d_{t}^{h^{2}} d t+e^{-\delta\left(T_{1} \wedge h\right)} V\left(U_{T_{1} \wedge h}^{\pi^{h^{2}}}\right)\right] \geq V(x)-h^{2} .
$$

Let $u_{t}$ denote $d_{t}^{h^{2}}$ conditioned on $T_{1}>t$ and $\phi(x, t)=x e^{r t}+$ $\int_{0}^{t} e^{r(t-s)}\left(c-u_{s}\right) d s$. Using the same way as above, we get that

$$
\begin{aligned}
h+ & \frac{V(\phi(x, h))-V(x)}{h}-\frac{1-e^{-(\lambda+\delta) h}}{h} V(\phi(x, h)) \\
& +\frac{e^{-\lambda h}}{h} \int_{0}^{h} e^{-\delta t} u_{t} d t \\
& +\frac{1}{h} \int_{0}^{h} \lambda e^{-\lambda t}\left[\int_{0}^{t} e^{-\delta s} u_{s} d s\right. \\
& \left.+e^{-\delta t} \int_{0}^{\infty} V(\phi(x, t)-y) p(y) d y\right] d t \geq 0 .
\end{aligned}
$$

Notice that

$$
\lim _{h \rightarrow 0+} \varphi(x, h)=\lim _{h \rightarrow 0+} \phi(x, h)=x .
$$

Let

$$
\begin{aligned}
& D^{+} V(x)=\limsup _{s \rightarrow 0+} \frac{V(x+s)-V(x)}{s}, \\
& D^{-} V(x)=\liminf _{s \rightarrow 0+} \frac{V(x+s)-V(x)}{s} .
\end{aligned}
$$

We know that $D^{+} V(x)$ and $D^{-} V(x)$ are finite by Lipschitz continuity and monotonicity. We assume that

$$
\lim _{h \rightarrow 0+} u_{h}=d_{0} .
$$

Then we get

$$
\lim _{h \rightarrow 0+} \frac{\phi(x, h)-x}{h}=c-d_{0}+r x .
$$

Choose a sequence $\left\{h_{n}^{(1)}\right\}$ such that

$$
\lim _{n \rightarrow \infty} \frac{V\left(\phi\left(x, h_{n}^{(1)}\right)\right)-V(x)}{\phi\left(x, h_{n}^{(1)}\right)-x}=D^{-} V(x) .
$$

Then the limit is

$$
\begin{aligned}
\lim _{n \rightarrow \infty} & \frac{V\left(\phi\left(x, h_{n}^{(1)}\right)\right)-V(x)}{h_{n}^{(1)}} \\
& =\lim _{n \rightarrow \infty} \frac{\phi\left(x, h_{n}^{(1)}\right)-x}{h_{n}^{(1)}} \cdot \frac{V\left(\phi\left(x, h_{n}^{(1)}\right)\right)-V(x)}{\phi\left(x, h_{n}^{(1)}\right)-x} \\
& =\left(c-d_{0}+r x\right) D^{-} V(x) .
\end{aligned}
$$

Taking $h=h_{n}^{(1)}$ in (30) and letting $n \rightarrow \infty$, we get

$$
\begin{aligned}
& \left(c-d_{0}+r x\right) D^{-} V(x)-(\lambda+\delta) V(x) \\
& \quad+d_{0}+\lambda \int_{0}^{\infty} V(x-y) p(y) d y \geq 0 .
\end{aligned}
$$

Using the same method as above, we choose a sequence $\left\{h_{n}^{(2)}\right\}$ such that

$$
\lim _{n \rightarrow \infty} \frac{V\left(\varphi\left(x, h_{n}^{(2)}\right)\right)-V(x)}{\varphi\left(x, h_{n}^{(2)}\right)-x}=D^{+} V(x) .
$$

Then taking $u=d_{0}$ and $h=h_{n}^{(2)}$ in (28) and letting $n \rightarrow \infty$, we have

$$
\begin{gathered}
\left(c-d_{0}+r x\right) D^{+} V(x)-(\lambda+\delta) V(x)+d_{0} \\
+\lambda \int_{0}^{\infty} V(x-y) p(y) d y \leq 0 .
\end{gathered}
$$

From (37) and (39), we know that

$$
D^{+} V(x) \leq D^{-} V(x) .
$$

On the other hand

$$
D^{+} V(x) \geq D^{-} V(x) .
$$

So we have

$$
D^{+} V(x)=D^{-} V(x)=V^{\prime}(x+) .
$$

Therefore, the maximal dividend-value function is differentiable from the right.

For the sequence $\left\{h_{n}^{(1)}\right\}$ and $u=d_{0}$, inequality (28) holds and

$$
\lim _{n \rightarrow \infty} \frac{V\left(\varphi\left(x, h_{n}^{(1)}\right)\right)-V(x)}{h_{n}^{(1)}}=\left(c-d_{0}+r x\right) V^{\prime}(x+) .
$$

Then taking limit on both sides of inequalities (28) and (30), we find that

$$
\begin{aligned}
& \left(c-d_{0}+r x\right) V^{\prime}(x+)-(\lambda+\delta) V(x) \\
& \quad+\lambda \int_{0}^{\infty} V(x-y) p(y) d y+d_{0}=0 .
\end{aligned}
$$

From (28) we conclude that for any $d$

$$
\begin{aligned}
(c-d & +r x) V^{\prime}(x+)-(\lambda+\delta) V(x) \\
& +\lambda \int_{0}^{\infty} V(x-y) p(y) d y+d \leq 0 .
\end{aligned}
$$

Thus, we get the HJB equation for $V^{\prime}(x+)$

$$
\begin{aligned}
\max _{0 \leq d \leq \alpha}\left\{(c-d+r x) V^{\prime}(x+)-(\lambda+\delta) V(x)\right. \\
\left.+\lambda \int_{0}^{\infty} V(x-y) p(y) d y+d\right\}=0 .
\end{aligned}
$$


Lemma 4. When $x>0$, the maximal value function $V(x)$ is differentiable from the left and the left derivative $V^{\prime}(x-)$ satisfies HJB equation:

$$
\begin{array}{r}
\max _{0 \leq d \leq \alpha}\left\{(c-d+r x) V^{\prime}(x-)-(\lambda+\delta) V(x)\right. \\
\left.+\lambda \int_{0}^{\infty} V(x-y) p(y) d y+d\right\}=0 .
\end{array}
$$

Proof. Let $h$ be small enough such that

$$
\rho(x, h)=\left(x+\frac{c}{r}\right) e^{-r h}-\frac{c}{r}
$$

is positive. Changing the initial capital $x$ into $\rho(x, h)$ and using the dynamic programming principle, we obtain

$$
\begin{aligned}
& \frac{V(\tilde{\varphi}(x, h))-V(\rho(x, h))}{h}-\frac{1-e^{-(\lambda+\delta) h}}{h} V(\widetilde{\varphi}(x, h)) \\
& +\frac{e^{-\lambda h}}{h} \int_{0}^{h} e^{-\delta t} u d t \\
& +\frac{1}{h} \int_{0}^{h} \lambda e^{-\lambda t}\left[\int_{0}^{t} e^{-\delta s} u d s\right. \\
& \left.+e^{-\delta t} \int_{0}^{\infty} V(\widetilde{\varphi}(x, h)-y) p(y) d y\right] d t \leq 0, \\
& h+\frac{V(\tilde{\phi}(x, h))-V(\rho(x, h))}{h}-\frac{1-e^{-(\lambda+\delta) h}}{h} V(\widetilde{\phi}(x, h)) \\
& +\frac{e^{-\lambda h}}{h} \int_{0}^{h} e^{-\delta t} u_{t} d t \\
& +\frac{1}{h} \int_{0}^{h} \lambda e^{-\lambda t}\left[\int_{0}^{t} e^{-\delta s} u_{s} d s\right. \\
& \left.+e^{-\delta t} \int_{0}^{\infty} V(\tilde{\phi}(x, t)-y) p(y) d y\right] d t \geq 0
\end{aligned}
$$

where

$$
\begin{aligned}
& \tilde{\varphi}(x, t)=\left[\left(x+\frac{c}{r}\right) e^{-r h}-\frac{c}{r}\right] e^{r t}+\int_{0}^{t} e^{r(t-s)}(c-u) d s, \\
& \tilde{\phi}(x, t)=\left[\left(x+\frac{c}{r}\right) e^{-r h}-\frac{c}{r}\right] e^{r t}+\int_{0}^{t} e^{r(t-s)}\left(c-u_{s}\right) d s .
\end{aligned}
$$

Let

$$
\begin{aligned}
& D_{+} V(x)=\limsup _{s \rightarrow 0-} \frac{V(x+s)-V(x)}{s}, \\
& D_{-} V(x)=\liminf _{s \rightarrow 0-} \frac{V(x+s)-V(x)}{s} .
\end{aligned}
$$

Using the same method as Lemma 3, we obtain

$$
D_{+} V(x)=D_{-} V(x)=V^{\prime}(x-),
$$

which implies the maximal dividend-value function which is differentiable from the left. Furthermore, the left derivative $V^{\prime}(x-)$ fulfills HJB equation:

$$
\begin{array}{r}
\max _{0 \leq d \leq \alpha}\left\{(c-d+r x) V^{\prime}(x-)-(\lambda+\delta) V(x)\right. \\
\left.+\lambda \int_{0}^{\infty} V(x-y) p(y) d y+d\right\}=0 .
\end{array}
$$

Theorem 5. The maximal value function $V(x)$ is continuously differentiable on $[0, \infty)$ and fulfills the following HJB equation:

$$
\begin{aligned}
\max _{0 \leq d \leq \alpha}\{( & (c-d+r x) V^{\prime}(x)-(\lambda+\delta) V(x) \\
& \left.+\lambda \int_{0}^{\infty} V(x-y) p(y) d y+d=0\right\},
\end{aligned}
$$

where $V^{\prime}(0)$ means the right derivative $V^{\prime}(0+)$.

Proof. From Lemmas 3 and 4, we know that function $V(x)$ is continuously differentiable and fulfills the HJB equation (54).

\section{Optimal Dividend Strategy}

Since $V(x)$ is increasing, concave and converges to $\alpha / \delta$, there exists a finite constant:

$$
b^{*}=\inf \left\{x: V^{\prime}(x) \leq 1\right\}
$$

such that

$$
\begin{aligned}
& V^{\prime}(x)>1, \quad \text { if } x<b^{*}, \\
& V^{\prime}(x) \leq 1, \quad \text { if } x \geq b^{*} .
\end{aligned}
$$

From Theorem 5, we construct a strategy $\pi^{*}=\left\{d_{t}^{*}, Z_{t}^{d^{*}}\right\}$ as follows:

$$
d_{t+}^{*}= \begin{cases}0, & \text { if } U_{t}^{\pi^{*}}<b^{*} \\ \alpha, & \text { if } U_{t}^{\pi^{*}} \geq b^{*}\end{cases}
$$

$Z_{t}^{d^{*}}$ is the corresponding capital injection process. This policy is a double level strategy with a lower barrier at zero and an upper threshold at level $b^{*}$. Whenever the surplus is between 0 and $b^{*}$, no dividends are paid and no capitals are injected; whenever the surplus is at or above $b^{*}$, dividends will be paid at the maximal rate $\alpha$, but no capitals are injected; whenever the surplus is below 0 , a required amount of the capital which are equal to the amount of the deficit are injected immediately. Figure 1 gives an intuitional description for the strategy $\pi^{*}$.

Theorem 6. The policy $\pi^{*}=\left\{d_{t}^{*}, Z_{t}^{d^{*}}\right\}$ which is given by (57) is optimal among all admissible policies.

Proof. First of all, the policy $\pi^{*}=\left\{d_{t}^{*}, Z_{t}^{d^{*}}\right\}$ is an admissible strategy. Let $V_{*}(x)$ denote the corresponding value function. 


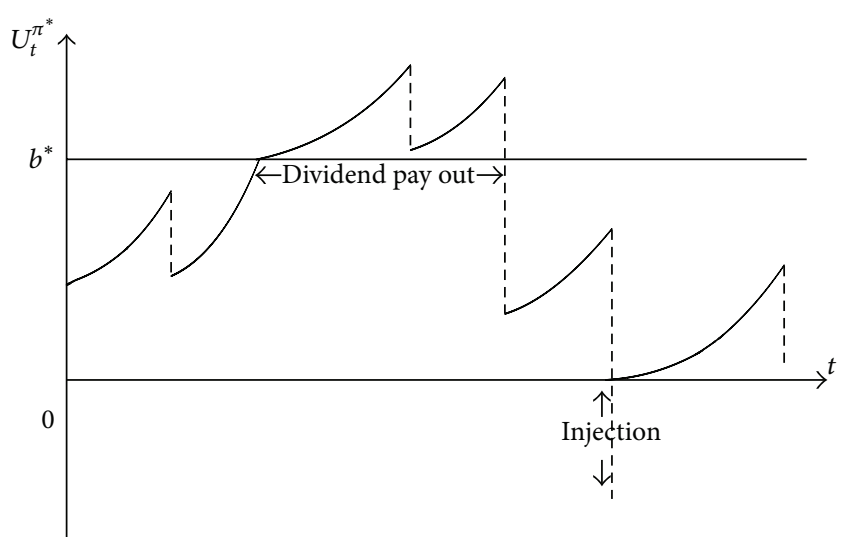

FIGURE 1: A typical sample path of the surplus process with strategy $\pi^{*}$.

By an application of Itô's formula to $e^{-\delta t} V\left(U_{t}^{\pi^{*}}\right)$, it can be verified that

$$
\begin{aligned}
& e^{-\delta t} V\left(U_{t}^{\pi^{*}}\right) \\
& =V(x)+\int_{0}^{t} e^{-\delta s}\left[\left(c-d_{s}^{\pi^{*}}+r U_{s-}^{\pi^{*}}\right)\right. \\
& \left.\times V^{\prime}\left(U_{s-}^{\pi^{*}}\right)-\delta V\left(U_{s-}^{\pi^{*}}\right)\right] d s \\
& +\sum_{\substack{0<s \leq t \\
U_{s-}^{\pi^{*}} \neq U_{s}^{\pi^{*}}}} e^{-\delta s}\left[V\left(U_{s}^{\pi^{*}}\right)-V\left(U_{s-}^{\pi^{*}}\right)\right] .
\end{aligned}
$$

Note that the injections occur only at the claim times. Let $z_{i}^{\pi^{*}}$ denote the amount of injected capital at the occurrence time of the $i$ th claim. If $U_{T_{i}-}^{\pi^{*}}-X_{i} \geq 0$, no capitals are injected, that is, $z_{i}^{\pi^{*}}=0$. If $U_{T_{i}-}^{\pi^{*}}-X_{i}<0, z_{i}^{\pi^{*}}=-\left(U_{T_{i}-}^{\pi^{*}}-X_{i}\right)$ and $U_{T_{i}}^{\pi^{*}}=$ $U_{T_{i^{-}}}^{\pi^{*}}-X_{i}+z_{i}^{\pi^{*}}=0$. For both cases, the equality

$$
V\left(U_{T_{i}}^{\pi^{*}}\right)=V\left(U_{T_{i}{ }^{-}}^{\pi^{*}}-X_{i}\right)+\kappa z_{i}^{\pi^{*}}
$$

holds. Since $U_{s-}^{\pi^{*}} \neq U_{s}^{\pi^{*}}$ only at the occurrence time of a claim, then

$$
\begin{aligned}
& \sum_{\substack{0<s \leq t \\
U_{s-}^{\pi^{*}} \neq U_{s}^{\pi^{*}}}} e^{-\delta s}\left[V\left(U_{s}^{\pi^{*}}\right)-V\left(U_{s-}^{\pi^{*}}\right)\right] \\
= & \sum_{i=1}^{N_{t}} e^{-\delta T_{i}}\left[V\left(U_{T_{i^{-}}}^{\pi^{*}}-X_{i}+z_{i}^{\pi^{*}}\right)-V\left(U_{T_{i}-}^{\pi^{*}}\right)\right] \\
= & \sum_{i=1}^{N_{t}} e^{-\delta T_{i}}\left[V\left(U_{T_{i}^{-}}^{\pi^{*}}-X_{i}\right)-V\left(U_{T_{i}}^{\pi^{*}}\right)\right]+\kappa \sum_{i=1}^{N_{t}} e^{-\delta T_{i}} z_{i}^{\pi^{*}} \\
= & \sum_{i=1}^{N_{t}} e^{-\delta T_{i}}\left[V\left(U_{T_{i^{-}}}^{\pi^{*}}-X_{i}\right)-V\left(U_{T_{i^{-}}}^{\pi^{*}}\right)\right]+\kappa \int_{0}^{t} e^{-\delta s} d Z_{s}^{d^{*}} .
\end{aligned}
$$

Taking expectations in (58), we obtain

$$
\begin{aligned}
& E\left[e^{-\delta t} V\left(U_{t}^{\pi^{*}}\right)\right] \\
& =V(x)+E \int_{0}^{t} e^{-\delta s}\left[\left(c-d_{s}^{\pi^{*}}+r U_{s-}^{\pi^{*}}\right) V^{\prime}\left(U_{s-}^{\pi^{*}}\right)\right. \\
& \left.\quad-\delta V\left(U_{s-}^{\pi^{*}}\right)\right] d s \\
& +\kappa E \int_{0}^{t} e^{-\delta s} d Z_{s}^{d^{*}} \\
& \quad+E \sum_{i=1}^{N_{t}} e^{-\delta T_{i}}\left[V\left(U_{T_{i}-}^{\pi^{*}}-X_{i}\right)-V\left(U_{T_{i}-}^{\pi^{*}}\right)\right] .
\end{aligned}
$$

Note that

$$
\begin{aligned}
& \sum_{i=1}^{N_{t}} e^{-\delta T_{i}}\left[V\left(U_{T_{i}-}^{\pi^{*}}-X_{i}\right)-V\left(U_{T_{i}}^{\pi^{*}}\right)\right] \\
& \quad-\lambda \int_{0}^{t} e^{-\delta s} \int_{0}^{\infty}\left[V\left(U_{s^{-}}^{\pi^{*}}-y\right)-V\left(U_{s^{-}}^{\pi^{*}}\right)\right] p(y) d y d s
\end{aligned}
$$

is a martingale with zero-expectation. Then

$$
\begin{aligned}
& E\left[e^{-\delta t} V\left(U_{t}^{\pi^{*}}\right)\right] \\
& =V(x)+E \int_{0}^{t} e^{-\delta s}\left[\left(c-d_{s}^{\pi^{*}}+r U_{s-}^{\pi^{*}}\right) V^{\prime}\left(U_{s-}^{\pi^{*}}\right)\right. \\
& -(\lambda+\delta) V\left(U_{s^{-}}^{\pi^{*}}\right) \\
& \left.+\lambda \int_{0}^{\infty} V\left(U_{s^{-}}^{\pi^{*}}-y\right) p(y) d y\right] d s \\
& +\kappa E \int_{0}^{t} e^{-\delta s} d Z_{s}^{d^{*}} .
\end{aligned}
$$

By HJB equation (54), we find that

$$
\begin{gathered}
\left(c-d_{s}^{\pi^{*}}+r U_{s-}^{\pi^{*}}\right) V^{\prime}\left(U_{s^{-}}^{\pi^{*}}\right)-(\lambda+\delta) V\left(U_{s-}^{\pi^{*}}\right) \\
+\lambda \int_{0}^{U_{s-}^{\pi^{*}}} V\left(U_{s^{-}}^{\pi^{*}}-y\right) p(y) d y=-d_{s}^{\pi^{*}} .
\end{gathered}
$$

Thus, we gather that

$$
\begin{gathered}
E\left[e^{-\delta t} V\left(U_{t}^{\pi^{*}}\right)\right]+E \int_{0}^{t} e^{-\delta s} d_{s}^{\pi^{*}} d s \\
-\kappa E \int_{0}^{t} e^{-\delta s} d Z_{s}^{d^{*}}=V(x) .
\end{gathered}
$$

Taking limit in $t \rightarrow \infty$, by the boundedness of $V$, we obtain that

$$
V_{*}(x)=E\left[\int_{0}^{\infty} e^{-\delta s} d_{s}^{\pi^{*}} d s-\kappa \int_{0}^{\infty} e^{-\delta s} d Z_{s}^{d^{*}}\right]=V(x) .
$$

This shows that the policy $\pi^{*}$ is the optimal strategy. 


\section{Concluding Remarks}

In summary, this paper gives the optimal strategy which maximizes the discounted dividend payments minus the penalized discounted capital injections for a risk model including a constant force of interest. The optimal dividend strategy is a threshold strategy for the case of the dividend payout rate which is bounded by some positive constant, and the optimal injection strategy is to inject capitals immediately to make the company's assets back to zero when the surplus of the company becomes negative.

We also wish to point out that further research is needed. Further extensions of the analysis of the problems in the paper could remove the restriction on the dividend payout rate. We hope that this open problem can be addressed in future research.

\section{Acknowledgments}

The authors would like to thank the referees for their helpful comments. The research was supported by National Natural Science Foundation of China (Grant nos. 11201271, 11126093, and 71071088).

\section{References}

[1] S. Asmussen and M. Taksar, "Controlled diffusion models for optimal dividend pay-out," Insurance: Mathematics and Economics, vol. 20, no. 1, pp. 1-15, 1997.

[2] H. U. Gerber, "Entscheidungskriterien für den zusammengesetzten Poisson-Prozess," Mitteilungen der Vereinigung Schweizerischer Versicherungsmathematiker, vol. 69, pp. 185-228, 1969.

[3] P. Azcue and N. Muler, "Optimal reinsurance and dividend distribution policies in the Cramér-Lundberg model," Mathematical Finance, vol. 15, no. 2, pp. 261-308, 2005.

[4] H. Schmidli, Stochastic Control in Insurance, Springer, London, UK, 2008.

[5] H. Albrecher and S. Thonhauser, "Optimal dividend strategies for a risk process under force of interest," Insurance: Mathematics and Economics, vol. 43, no. 1, pp. 134-149, 2008.

[6] F. Avram, Z. Palmowski, and M. R. Pistorius, "On the optimal dividend problem for a spectrally negative levy process," Annals of Applied Probability, vol. 17, no. 1, pp. 156-180, 2007.

[7] R. L. Loeffen, "On optimality of the barrier strategy in de Finetti's dividend problem for spectrally negative Lévy processes," Annals of Applied Probability, vol. 18, no. 5, pp. 16691680, 2008.

[8] S. E. Shreve, J. P. Lehoczky, and D. P. Gaver, "Optimal consumption for general diffusions with absorbing and reflecting barriers," SIAM Journal on Control and Optimization, vol. 22, no. 1, pp. 55-75, 1984.

[9] A. Løkka and M. Zervos, "Optimal dividend and issuance of equity policies in the presence of proportional costs," Insurance: Mathematics and Economics, vol. 42, no. 3, pp. 954-961, 2008.

[10] N. Kulenko and H. Schmidli, "Optimal dividend strategies in a Cramér-Lundberg model with capital injections," Insurance: Mathematics and Economics, vol. 43, no. 2, pp. 270-278, 2008.

[11] M. Jeanblanc-Picqué and A. N. Shiryaev, "Optimization of the flow of dividends," Russian Mathematical Surveys, vol. 50, pp. 257-277, 1995.
[12] W. H. Fleming and H. M. Soner, Controlled Markov Processes and Viscosity Solutions, Springer, New York, NY, USA, 1993.

[13] J. Huang, G. Wang, and Z. Wu, "Optimal premium policy of an insurance firm: full and partial information," Insurance: Mathematics and Economics, vol. 47, no. 2, pp. 208-215, 2010. 


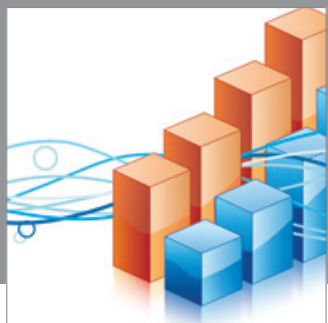

Advances in

Operations Research

mansans

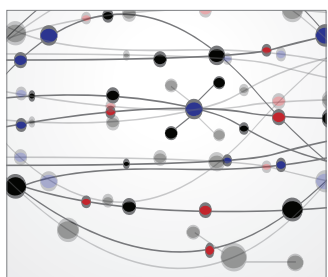

The Scientific World Journal
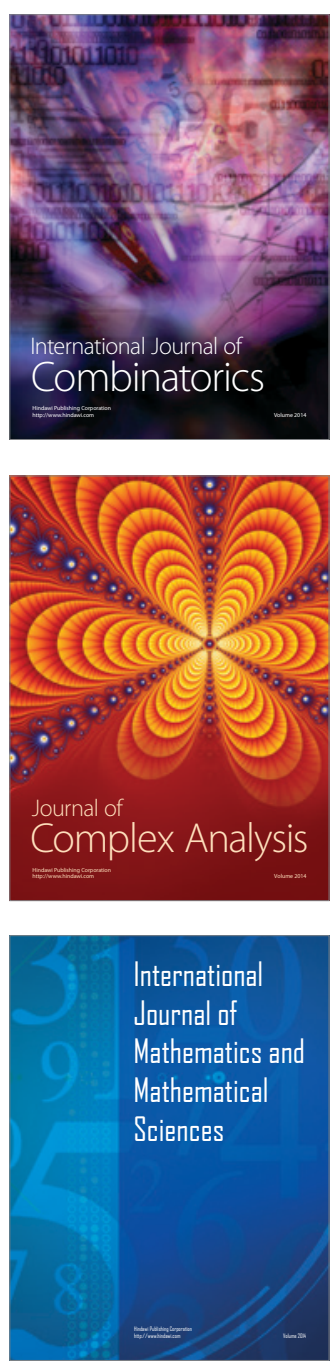
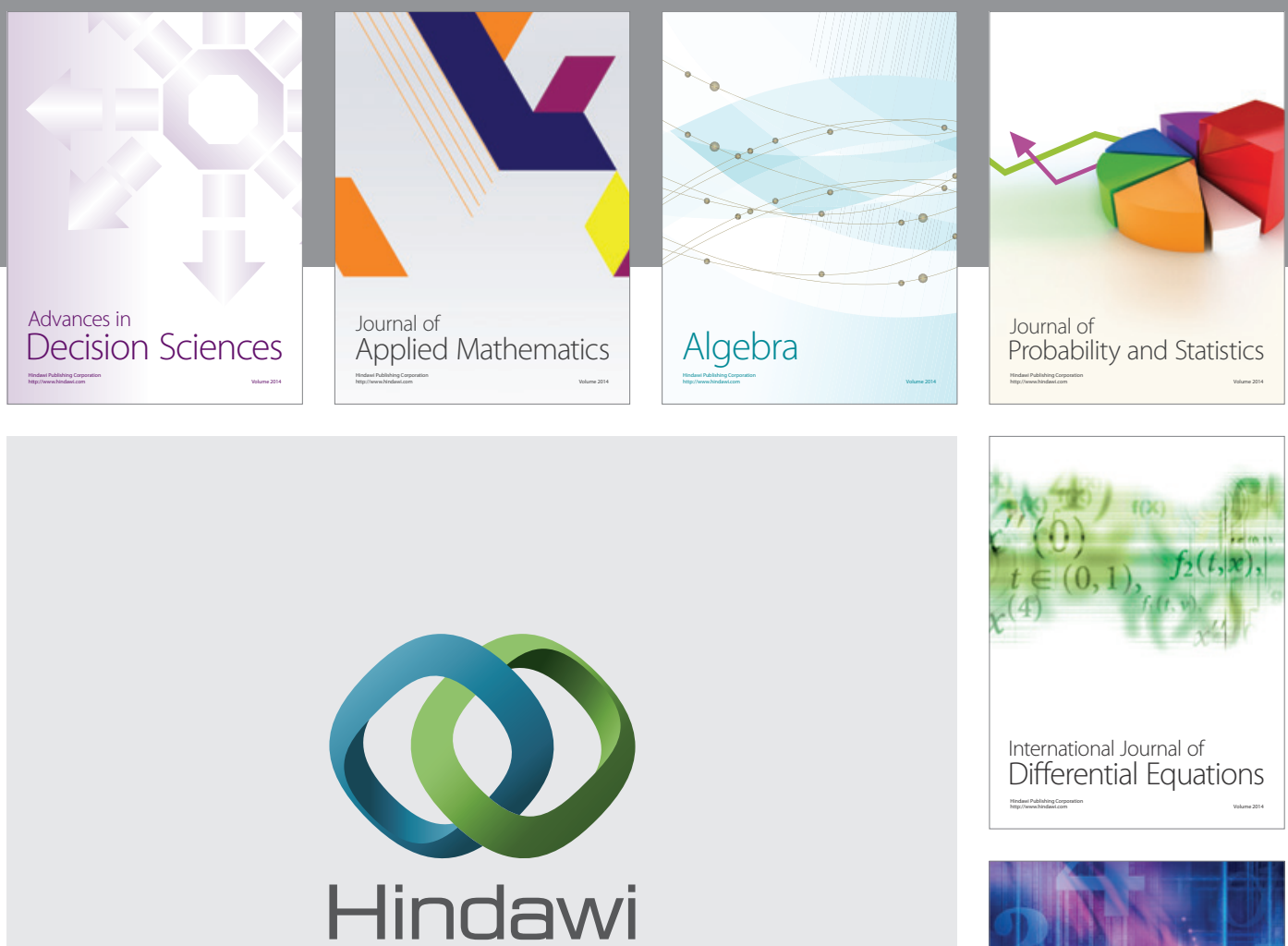

Submit your manuscripts at http://www.hindawi.com
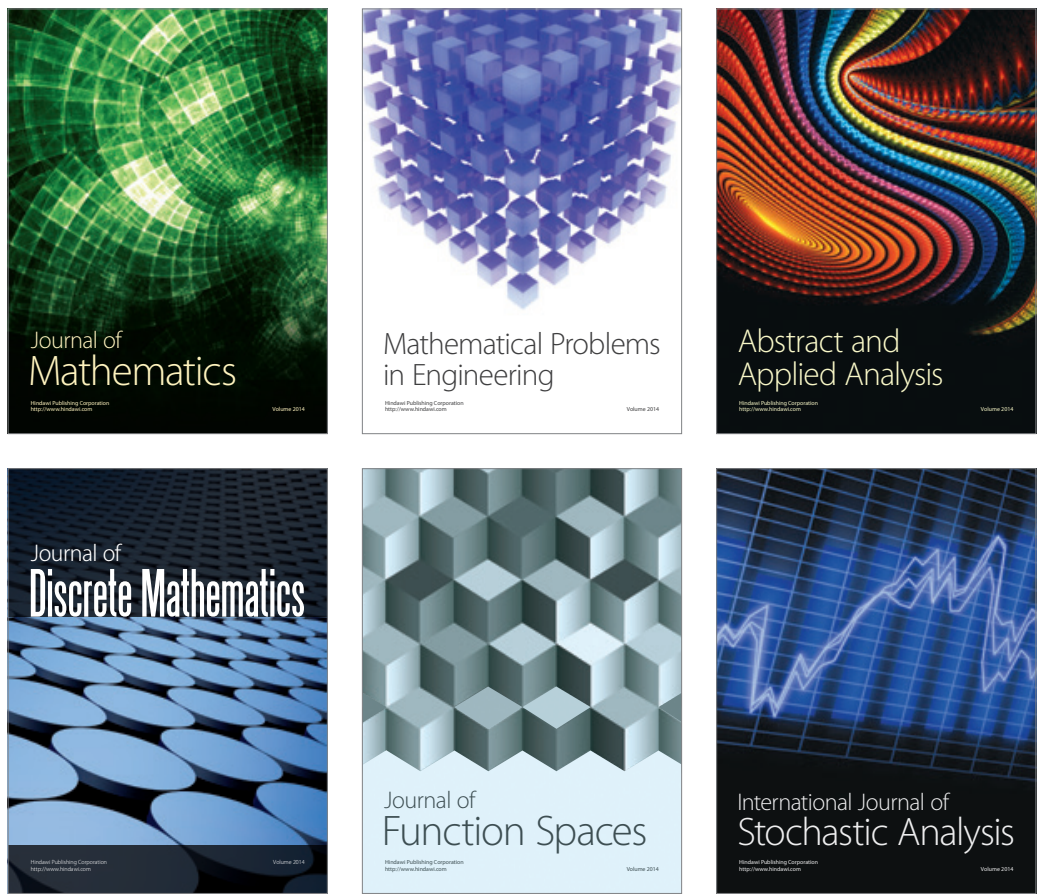

Journal of

Function Spaces

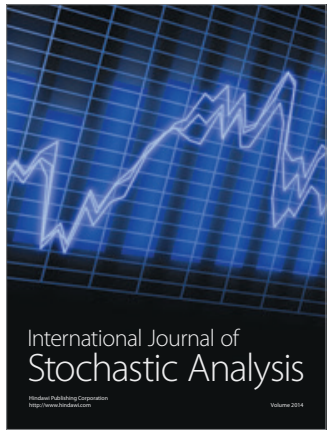

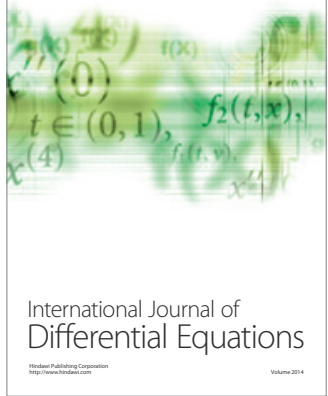
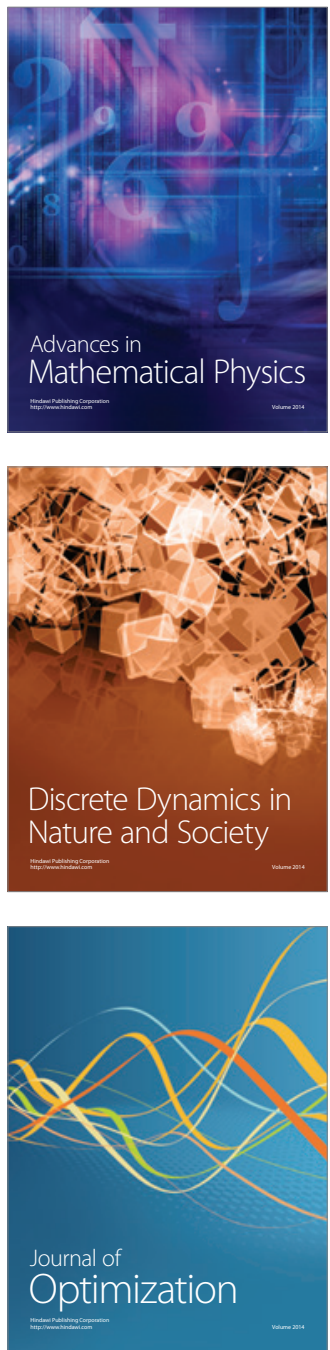